\title{
Cardiovascular safety of nintedanib in subgroups by cardiovascular risk at baseline in the TOMORROW and INPULSIS trials
}

\author{
Imre Noth ${ }^{1}$, Marlies Wijsenbeek ${ }^{2}$, Martin Kolb $\mathbb{1}^{3}$, Francesco Bonella ${ }^{4}$, \\ Lizette Moros ${ }^{5}$, Daniel Wachtlin ${ }^{6}$ and Tamera J. Corte
}

Affiliations: ${ }^{1}$ Division of Pulmonary and Critical Care Medicine, University of Virginia School of Medicine, Charlottesville, VA, USA. ${ }^{2}$ Dept of Pulmonary Medicine, Erasmus MC, University Medical Center, Rotterdam, The Netherlands. ${ }^{3}$ Dept of Respiratory Medicine, Pathology and Molecular Medicine, McMaster University, Hamilton, ON, Canada. ${ }^{4}$ Interstitial and Rare Lung Disease Unit, Ruhrlandklinik, University Hospital, University of Duisburg-Essen, Essen, Germany. ${ }^{5}$ Boehringer Ingelheim International $\mathrm{GmbH}$, Ingelheim am Rhein, Germany. ${ }^{6}$ Boehringer Ingelheim Pharma GmbH \& Co. KG, Ingelheim am Rhein, Germany. ${ }^{7}$ Royal Prince Alfred Hospital, Camperdown, Australia. ${ }^{8}$ University of Sydney, Sydney, Australia.

Correspondence: Imre Noth, Division of Pulmonary and Critical Care Medicine, University of Virginia School of Medicine, 1215 Lee Street, Charlottesville, VA 22908, USA. E-mail: IN2Cahscmail.mcc.virginia.edu

@ERSpublications

In pooled data from the randomised TOMORROW and INPULSIS trials in patients with IPF, the incidence rates of major adverse cardiovascular events were similar in the nintedanib and placebo groups both in patients with higher and lower cardiovascular risk http://bit.ly/2KUY8IP

Cite this article as: Noth I, Wijsenbeek M, Kolb M, et al. Cardiovascular safety of nintedanib in subgroups by cardiovascular risk at baseline in the TOMORROW and INPULSIS trials. Eur Respir J 2019; 54: 1801797 [https://doi.org/10.1183/13993003.01797-2018].

ABSTRACT Nintedanib is a tyrosine kinase inhibitor used to treat idiopathic pulmonary fibrosis (IPF). We investigated the cardiovascular safety of nintedanib using pooled data from the TOMORROW and INPULSIS trials.

Cardiovascular events were assessed post hoc in patients with a history of atherosclerotic cardiovascular disease (CVD) and/or one or more cardiovascular risk factors at baseline ("higher cardiovascular risk") and patients with no history of atherosclerotic CVD and no cardiovascular risk factors at baseline ("lower cardiovascular risk").

Incidence rates were calculated for 1231 patients ( $n=723$ nintedanib and $n=508$ placebo), of whom 89.9\% had higher cardiovascular risk. Incidence rates of major adverse cardiovascular events were similar in the nintedanib and placebo groups in patients with higher cardiovascular risk (3.88 (95\% CI 2.58-5.84) and 3.49 (95\% CI 2.10-5.79) per 100 patient-years, respectively) and lower cardiovascular risk (4.78 (95\% CI $1.54-14.82)$ and 5.37 (95\% CI $1.73-16.65)$ per 100 patient-years, respectively). Incidence rates of myocardial infarction in the nintedanib and placebo groups, respectively, were 3.03 (95\% CI 1.91-4.81) and 1.16 (95\% CI $0.48-2.79)$ per 100 patient-years in patients with higher cardiovascular risk and 1.59 (95\% CI 0.22-11.29) and 1.78 (95\% CI 0.25-12.64) per 100 patient-years in patients with lower cardiovascular risk. Incidence rates of other ischaemic heart disease in the nintedanib and placebo groups, respectively, were 1.85 (95\% CI 1.02-3.34) and 3.28 (95\% CI 1.94-5.54) per 100 patient-years in patients with higher cardiovascular risk and 0 and 1.80 (95\% CI $0.25-12.78)$ per 100 patient-years in patients with lower cardiovascular risk.

These data help to establish the cardiovascular safety profile of nintedanib in IPF.

This article has supplementary material available from erj.ersjournals.com

Copyright OERS 2019. This version is distributed under the terms of the Creative Commons Attribution NonCommercial Licence 4.0. 


\section{Introduction}

Idiopathic pulmonary fibrosis (IPF) is a progressive fibrosing interstitial lung disease characterised by decline in lung function and worsening dyspnoea [1]. IPF occurs more frequently in males and in ex-smokers, and typically presents in the sixth or seventh decade of life [1].

Cardiovascular diseases (CVDs) represent common comorbidities in patients with IPF [2-6]. Cardiovascular conditions associated with IPF include ischaemic heart disease (IHD), cerebrovascular disease and systemic arterial hypertension $[2,4]$. Cardiovascular risk is a continuum and is influenced by a number of risk factors. Guidelines for prevention of CVD issued by the European Society of Cardiology recommend an assessment of total cardiovascular risk in apparently healthy adults aged $>40$ years or those with relevant risk factors such as smoking, hypertension, cholesterol levels and diabetes [7].

Nintedanib is an approved treatment for IPF that reduces disease progression by reducing the rate of decline in forced vital capacity (FVC) [8]. In the latest international treatment guidelines for IPF, nintedanib received a conditional recommendation for use, indicating that it would be an appropriate choice for the majority of patients [4]. Nintedanib is an intracellular inhibitor of tyrosine kinases, including vascular endothelial growth factor, fibroblast growth factor and platelet-derived growth factor receptors $[9,10]$. Other tyrosine kinase inhibitors with different inhibition profiles have been associated with cardiovascular adverse events including pulmonary hypertension, left ventricular dysfunction and arterial thromboembolic events [11]. In the phase III INPULSIS trials, when broad search terms for myocardial infarction or other IHD were used, a higher proportion of patients in the nintedanib group than in the placebo group were found to have myocardial infarction (2.7\% (17 out of 638) versus $1.2 \%$ (five out of 423)), while a lower proportion had other IHD (1.7\% (11 out of 638 ) versus $3.1 \%$ (13 out of 423)) [12]. When narrow search terms were used, which took into account only those cases that were highly likely to represent myocardial infarction or IHD, 1.7\% (11 out of 638) of patients in the nintedanib group versus $0.5 \%$ (two out of 423 ) of patients in the placebo group had myocardial infarction and $1.6 \%$ (10 out of 638 ) of patients in the nintedanib group versus $3.1 \%$ (13 out of 423 ) of patients in the placebo group had other IHD (data on file). For two out of the 11 nintedanib-treated patients who had a myocardial infarction, this event was judged as drug related (data on file). The US and European Union prescribing information for nintedanib recommends caution when treating patients at higher cardiovascular risk, including those with coronary artery disease $[13,14]$.

We assessed the effect of cardiovascular risk at baseline on the cardiovascular safety profile of nintedanib in patients with IPF using pooled data from the randomised placebo-controlled phase II TOMORROW trial and phase III INPULSIS trials.

\section{Methods}

Data were pooled from the nintedanib $150 \mathrm{mg}$ twice daily and placebo groups of the phase II TOMORROW trial and the phase III INPULSIS trials. The designs and results of these trials have been reported [8, 15]. Briefly, eligible patients were $\geqslant 40$ years of age with a diagnosis of IPF, FVC $\geqslant 50 \%$ predicted and diffusing capacity of the lung for carbon monoxide $30-79 \%$ predicted. Patients with a recent history of myocardial infarction (previous 6 months), unstable angina (previous month) or stroke (previous year) were excluded. In each trial, patients were randomised to receive nintedanib or placebo for 52 weeks.

Post hoc subgroup analyses were conducted in patients with a history of atherosclerotic CVD and/or one or more cardiovascular risk factors at baseline ("higher cardiovascular risk") and patients with no history of atherosclerotic CVD and no cardiovascular risk factors at baseline ("lower cardiovascular risk"). "History of atherosclerotic CVD" was assessed based on the comorbidities reported by the patient and provided in case report forms. These were coded according to preferred terms in the Medical Dictionary for Regulatory Activities (MedDRA) version 16.1. For the purposes of this analysis, "atherosclerotic CVD" was identified based on the subordinate Standardised MedDRA Queries (SMQs) "myocardial infarction" and "other IHD" within the SMQ "IHD", the subordinate SMQ "ischaemic cerebrovascular conditions" within the SMQ "cerebrovascular disorders", and the MedDRA preferred terms "peripheral arterial occlusive disease", "ischaemia" and "arteriosclerosis" (table 1). "Cardiovascular risk factors" were defined based on the SMQs "hypertension" (including metabolic syndrome, essential hypertension (primary/ essential/idiopathic hypertension), hypertensive cardiomyopathy and hypertensive heart disease), "dyslipidaemia" (including hypercholesterolaemia, hypertriglyceridaemia, combined hyperlipidaemia and secondary dyslipidaemia), as well as body mass index (BMI) $>30 \mathrm{~kg} \cdot \mathrm{m}^{-2}$, current/ex-smoker and diabetes at baseline (table 1). These criteria for identifying patients at higher and lower cardiovascular risk were chosen arbitrarily, but based on cardiovascular risk factors identified by professional associations [7]. It was not possible to use a published cardiovascular risk scoring system given the data that were collected on the patients who participated in the trials. 
TABLE 1 Definitions for atherosclerotic cardiovascular disease (CVD) and cardiovascular risk factors

Atherosclerotic CVD

Cardiovascular risk factors
Myocardial infarction (subordinate SMQ)

Other ischaemic heart disease (subordinate SMQ)

Ischaemic cerebrovascular conditions (subordinate SMQ)

Peripheral arterial occlusive disease (MedDRA preferred term) Ischaemia (MedDRA preferred term)

Arteriosclerosis (MedDRA preferred term)

Hypertension (SMQ)

Dyslipidaemia (SMQ)

Body mass index $>30 \mathrm{~kg} \cdot \mathrm{m}^{-2}$

Current/ex-smoker

Diabetes

MedDRA: Medical Dictionary for Regulatory Activities; SMQ: Standardised MedDRA Query.

Baseline cardiovascular therapies were classified according to World Health Organization Anatomical Therapeutic Chemical coding. Incidence rates (per 100 patient-years) of adverse events of myocardial infarction, other IHD and major adverse cardiovascular events (MACE) were analysed. Myocardial infarction and other IHD were based on one or more events in the subordinate SMQs "myocardial infarction" and "other IHD", respectively. MACE was based on one or more events based on fatal adverse events included in the MedDRA system organ classes "cardiac disorders" and "vascular disorders", fatal and nonfatal events in the subordinate SMQ "myocardial infarction", stroke based on selected preferred terms from the subordinate SMQs "haemorrhagic cerebrovascular conditions" and "ischaemic cerebrovascular conditions", and the MedDRA preferred terms "sudden death", "cardiac death" and "sudden cardiac death".

Adverse events with onset after the first dose and up to 14 days (in the TOMORROW trial) or 28 days (in the INPULSIS trials) after the last dose of study drug were included in the analyses. Analyses were descriptive and based on patients who received one or more doses of study drug.

\section{Results}

\section{Cardiovascular risk at baseline}

1231 patients were included in this analysis (723 treated with nintedanib $150 \mathrm{mg}$ twice daily and 508 treated with placebo). At baseline, 1107 patients (89.9\%) had higher cardiovascular risk and 124 patients (10.1\%) had lower cardiovascular risk, according to the aforementioned definitions. The proportion of patients with higher cardiovascular risk was similar in the nintedanib and placebo groups (table 2). Patients at higher cardiovascular risk had higher weight and BMI, and a greater proportion were male (table 3). Baseline characteristics were generally similar in the nintedanib and placebo groups within each cardiovascular risk subgroup, although the proportion of Asians was higher in the nintedanib group. Of patients with higher cardiovascular risk at baseline, $22 \%$ had a history of atherosclerotic CVD. The most common form of atherosclerotic CVD was coronary artery disease (supplementary table S1). Of patients with higher cardiovascular risk, $65.5 \%$ had one or two cardiovascular risk factors (figure 1). Current/ ex-smoker was the most common risk factor (79.7\%), followed by hypertension (47.9\%), dyslipidaemia (35.7\%), BMI $>30 \mathrm{~kg} \cdot \mathrm{m}^{-2}(30.6 \%)$ and diabetes (22.6\%) (figure 2). The proportions of patients with each risk factor were similar in the nintedanib and placebo groups. Four patients $(0.6 \%)$ in the nintedanib group and four patients $(0.9 \%)$ in the placebo group had no cardiovascular risk factors (as defined in this analysis) but had a history of atherosclerotic CVD. In patients with higher cardiovascular risk, $66.2 \%$ were

TABLE 2 Cardiovascular risk at baseline

\begin{tabular}{lcc} 
& Nintedanib & Placebo \\
\hline Subjects & 723 & 508 \\
Higher cardiovascular risk & $656(90.7)$ & $451(88.8)$ \\
With history of atherosclerotic CVD & $143(19.8)$ & $102(20.1)$ \\
$\quad$ Without history of atherosclerotic CVD & $513(70.9)$ & $349(68.7)$ \\
Lower cardiovascular risk & $67(9.3)$ & $57(11.2)$ \\
\hline
\end{tabular}

Data are presented as $\mathrm{n}$ or $\mathrm{n}(\%)$. CVD: cardiovascular disease. 
TABLE 3 Baseline characteristics by cardiovascular risk at baseline

\begin{tabular}{|c|c|c|c|c|}
\hline & \multicolumn{2}{|c|}{ Higher cardiovascular risk } & \multicolumn{2}{|c|}{ Lower cardiovascular risk } \\
\hline & Nintedanib & Placebo & Nintedanib & Placebo \\
\hline Subjects & 656 & 451 & 67 & 57 \\
\hline Age years & $66.6 \pm 8.1$ & $66.9 \pm 7.9$ & $65.2 \pm 8.0$ & $64.3 \pm 8.6$ \\
\hline Male & $530(80.8)$ & $364(80.7)$ & $42(62.7)$ & 33 (57.9) \\
\hline \multicolumn{5}{|l|}{ Race } \\
\hline White & 385 (58.7) & $291(64.5)$ & 36 (53.7) & $22(38.6)$ \\
\hline Asian & $193(29.4)$ & $117(25.9)$ & 25 (37.3) & $31(54.4)$ \\
\hline Weight kg & $79.7 \pm 16.4$ & $79.8 \pm 15.7$ & $69.5 \pm 13.4$ & $67.3 \pm 13.9$ \\
\hline BMI $\mathrm{kg} \cdot \mathrm{m}^{-2}$ & $28.2 \pm 4.6$ & $27.9 \pm 4.5$ & $25.2 \pm 2.9$ & $24.7 \pm 3.0$ \\
\hline$<18.5 \mathrm{~kg} \cdot \mathrm{m}^{-2}$ & $3(0.5)$ & $3(0.7)$ & $1(1.5)$ & $2(3.5)$ \\
\hline$\geqslant 18.5-<25 \mathrm{~kg} \cdot \mathrm{m}^{-2}$ & $165(25.2)$ & $131(29.0)$ & $31(46.3)$ & $29(50.9)$ \\
\hline$\geqslant 25-\leqslant 30 \mathrm{~kg} \cdot \mathrm{m}^{-2}$ & $286(43.6)$ & $180(39.9)$ & $35(52.2)$ & $26(45.6)$ \\
\hline$>30 \mathrm{~kg} \cdot \mathrm{m}^{-2}$ & $202(30.8)$ & $137(30.4)$ & 0 & 0 \\
\hline \multicolumn{5}{|l|}{ Smoking history } \\
\hline Current smoker & $31(4.7)$ & 24 (5.3) & 0 & 0 \\
\hline Ex-smoker & 493 (75.2) & $334(74.1)$ & 0 & 0 \\
\hline Never-smoker & $132(20.1)$ & $93(20.6)$ & $67(100.0)$ & 57 (100.0) \\
\hline Diabetes & 149 (22.7) & $101(22.4)$ & 0 & 0 \\
\hline Dyslipidaemia & $240(36.6)$ & 155 (34.4) & 0 & 0 \\
\hline Hypertension & 310 (47.3) & $220(48.8)$ & 0 & 0 \\
\hline FVC $\%$ pred & $79.6 \pm 17.7$ & $79.8 \pm 18.4$ & $80.0 \pm 17.4$ & $78.5 \pm 16.1$ \\
\hline
\end{tabular}

Data are presented as $n$, mean \pm SD or $n(\%)$. BMI: body mass index; FVC: forced vital capacity.

receiving a therapy for CVD, 36.4\% were receiving a lipid-modifying agent (e.g. a statin) and 30.9\% were receiving an antithrombotic agent (e.g. acetylsalicylic acid) (figure 3 and supplementary table S2). The proportions of patients receiving specific cardiovascular therapies were generally similar in the nintedanib and placebo groups.

\section{Cardiovascular adverse events}

In the whole pooled population, myocardial infarction, other IHD and MACE adverse events were reported in $2.0 \%, 2.1 \%$ and $3.6 \%$ of patients, respectively. In patients with higher cardiovascular risk at baseline, the incidence rate of myocardial infarction was 3.03 (95\% CI 1.91-4.81) per 100 patient-years in the nintedanib group and 1.16 (95\% CI 0.48-2.79) per 100 patient-years in the placebo group. The incidence rate of other IHD was 1.85 (95\% CI 1.02-3.34) per 100 patient-years in the nintedanib group and 3.28 (95\% CI 1.94-5.54) per 100 patient-years in the placebo group (figure 4). In patients with lower cardiovascular risk at baseline, the incidence rates of myocardial infarction and other IHD were similar in the nintedanib and placebo groups, but the number of patients and the number of events were very low (figure 4). The types of event reported are shown in supplementary table S3. The incidence rates of MACE were similar in the nintedanib and placebo groups, both in patients with higher cardiovascular risk at baseline (3.88 (95\% CI 2.58-5.84) and 3.49 (95\% CI 2.10-5.79) per 100 patient-years, respectively) and in patients with lower cardiovascular risk at baseline (4.78 (95\% CI 1.54-14.82) and 5.37 (95\% CI

FIGURE 1 Number of cardiovascular risk factors in patients with higher cardiovascular risk at baseline.

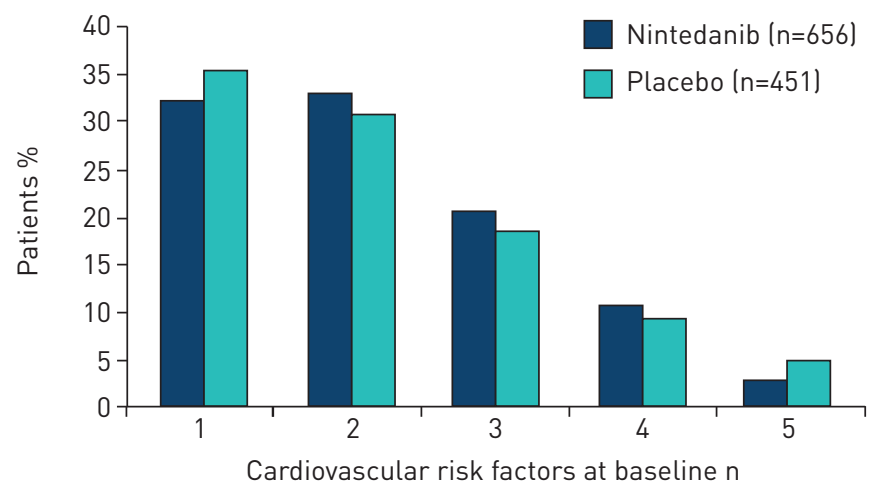


FIGURE 2 Frequency of cardiovascular risk factors in patients with higher cardiovascular risk at baseline.

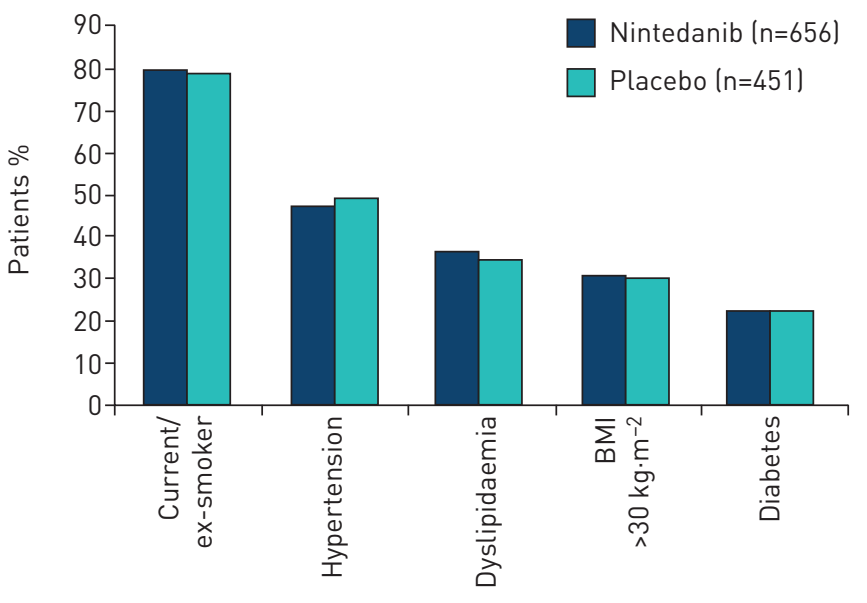

1.73-16.65) per 100 patient-years, respectively) (figure 4). The types of event reported are shown in supplementary table S4.

\section{Discussion}

We assessed the cardiovascular safety of nintedanib in subgroups by cardiovascular risk at baseline using pooled data from the TOMORROW and INPULSIS trials. Most patients (90\%) in these trials were defined as having higher cardiovascular risk at baseline, of which $78 \%$ had one or more cardiovascular risk factors but no history of atherosclerotic CVD. For the purpose of our analyses, cardiovascular risk factors were defined as hypertension, dyslipidaemia, BMI $>30 \mathrm{~kg} \cdot \mathrm{m}^{-2}$, current/ex-smoker and diabetes. Age and sex were not included as cardiovascular risk factors in our analyses, as IPF predominantly occurs in older males and this was reflected in the patient populations enrolled in these clinical trials. The majority of patients with higher cardiovascular risk at baseline were receiving a cardiovascular therapy, most commonly statins, and almost a third were receiving an antithrombotic agent.

Irrespective of cardiovascular risk at baseline, the incidence rate of MACE was low $(<5$ per 100 patientyears), and was similar in the nintedanib and placebo groups. In patients with higher cardiovascular risk at baseline, the incidence rate of myocardial infarction was higher in the nintedanib group than in the placebo group, whereas the incidence rate of other IHD was lower in the nintedanib group than in the placebo group. The clinical significance of these findings is unknown. In patients with lower cardiovascular risk, the incidence rates of myocardial infarction and other IHD were similar between treatment groups, but the number of patients and the number of events were very low. Our data are

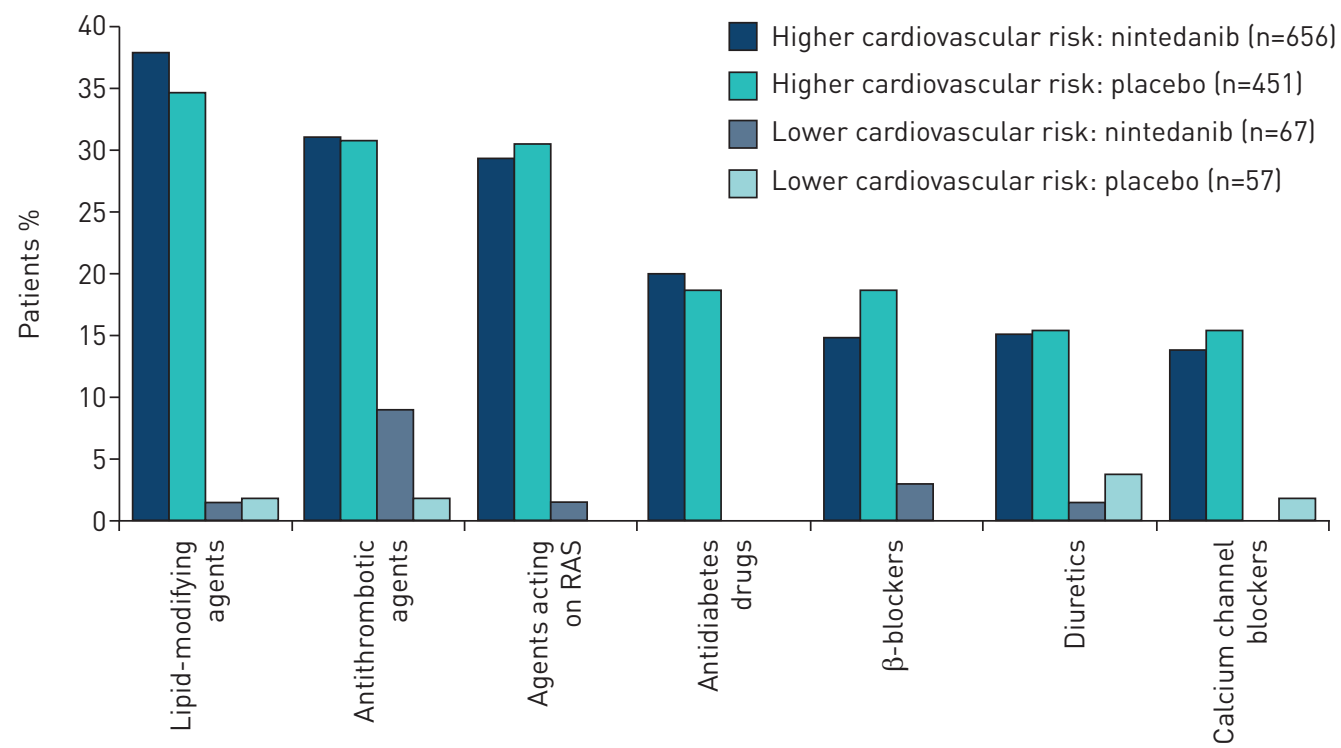

FIGURE 3 Baseline cardiovascular therapy by cardiovascular risk at baseline. RAS: renin-angiotensin system. 


\begin{tabular}{|c|c|c|c|c|}
\hline & $\begin{array}{c}\text { Patients } \\
\mathrm{n}\end{array}$ & $\begin{array}{l}\text { Patients with } \\
\geqslant 1 \text { events } \mathrm{n}(\%)\end{array}$ & $\begin{array}{l}\text { Rate per } 100 \\
\text { patient-years } \\
(95 \% \mathrm{CI})\end{array}$ & \\
\hline \multicolumn{5}{|c|}{$\begin{array}{l}\text { Myocardial infarction } \\
\text { Higher CV risk }\end{array}$} \\
\hline Nintedanib & 656 & $18(2.7)$ & $3.03(1.91-4.81)$ & \\
\hline Placebo & 451 & $5(1.1)$ & $1.16(0.48-2.79)$ & $\vdash$ \\
\hline \multicolumn{5}{|l|}{ Lower CV risk } \\
\hline Nintedanib & 67 & $1(1.5)$ & $1.59(0.22-11.29)$ & $\longrightarrow$ \\
\hline Placebo & 57 & $1(1.8)$ & $1.78(0.25-12.64)$ & $\longmapsto-$ \\
\hline \\
\hline Nintedanib & 656 & $11(1.7)$ & $1.85(1.02-3.34)$ & $\Omega$ \\
\hline Placebo & 451 & $14(3.1)$ & $3.28(1.94-5.54)$ & $\longrightarrow$ \\
\hline \multicolumn{5}{|l|}{ Lower CV risk } \\
\hline Nintedanib & 67 & $0(0)$ & 0.00 & \\
\hline Placebo & 57 & $1(1.8)$ & 1.80 (0.25-12.78) & \\
\hline \multicolumn{5}{|l|}{$\begin{array}{l}\text { MACE } \\
\text { Higher CV risk }\end{array}$} \\
\hline Nintedanib & 656 & 23 (3.5) & $3.88(2.58-5.84)$ & \\
\hline Placebo & 451 & $15(3.3)$ & $3.49(2.10-5.79)$ & \\
\hline \multicolumn{5}{|l|}{ Lower CV risk } \\
\hline Nintedanib & 67 & $3(4.5)$ & $4.78(1.54-14.82)$ & $\longmapsto \longmapsto$ \\
\hline \multirow[t]{2}{*}{ Placebo } & 57 & $3(5.3)$ & $5.37(1.73-16.65)$ & $\longmapsto \longrightarrow$ \\
\hline & & & & $\begin{array}{lllllllllllllllllll}0 & 1 & 2 & 3 & 4 & 5 & 6 & 7 & 8 & 9 & 10 & 11 & 12 & 13 & 14 & 15 & 16 & 17 & 18\end{array}$ \\
\hline
\end{tabular}

FIGURE 4 Incidence rates of myocardial infarction, other ischaemic heart disease (IHD) and major adverse cardiovascular (CV) events (MACE) by CV risk at baseline. See main text for more details of MACE.

consistent with data from INPULSIS-ON, the open-label extension of the INPULSIS trials, which showed a similarly low incidence rate of MACE in patients treated with nintedanib in both trials (3.19 (95\% CI 2.27-4.49) per 100 patient-years) after a mean \pm SD total exposure to nintedanib of $40.7 \pm 14.6$ months [16]. The incidence rate of myocardial infarction in this analysis was 0.77 (95\% CI 0.39-1.54) per 100 patient-years. Taken together, these data do not suggest cardiovascular safety concerns over the use of nintedanib in patients with IPF.

Patients with IPF treated with nintedanib in clinical practice appear to have cardiac adverse event profiles that are similar to those observed in clinical trials despite having more impaired lung function and more comorbidities [17-22]. However, such comparisons should be made with caution given the different methodologies used to collect the data. Data from post-marketing surveillance in the USA conducted for 1 year after the launch of nintedanib as a treatment for IPF showed incidence rates for MACE and myocardial infarction of 2.9 and 1.0 per 100 patient-years, respectively [20]. Scant data are available on rates of myocardial infarction in patients with IPF, but the incidence rate of myocardial infarction in patients treated with nintedanib in the TOMORROW and INPULSIS trials is similar to that reported in an analysis of US healthcare claims data from 9286 patients with IPF prior to the availability of antifibrotic therapy (2.2 per 100 patient-years) [2].

Our analyses have a number of limitations: patients were not randomised by cardiovascular risk at baseline, no data on cardiovascular therapy use prior to the trials were available, and analyses were conducted post hoc, with no formal statistical tests performed, and not based on risk calculations. The definition used for "high cardiovascular risk" was broad, including patients with even a single cardiovascular risk factor. A substantial proportion of patients in the "low cardiovascular risk" subgroup were males or aged $>65$ years, which are established risk factors for CVD. Patients with a recent history of myocardial infarction (previous 6 months), unstable angina (previous month) or stroke (previous year) were excluded from these trials. Caution should be exercised in the interpretation of these data given the uncertainty arising from sampling variability, especially for the small subgroup of patients with no cardiovascular risk factors at baseline, and the limitations in attributing observed differences in event rates to randomised treatment, use of cardiovascular medications or patient characteristics.

In conclusion, post hoc analyses of pooled data from the TOMORROW and INPULSIS trials suggest that the incidence of MACE was similar between patients treated with nintedanib and placebo irrespective of 
cardiovascular risk at baseline. The incidence of myocardial infarction was higher in the nintedanib group than in the placebo group among patients with higher cardiovascular risk, whereas the incidence rate of other IHD was lower in the nintedanib group than in the placebo group. Overall, the incidence of myocardial infarction was similar in nintedanib-treated patients in the TOMORROW and INPULSIS trials as in epidemiological data from patients with IPF not treated with nintedanib. These data help to establish the cardiovascular safety profile of nintedanib in patients with IPF. Observational and pharmacovigilance studies will continue to provide additional data on cardiovascular events in patients with IPF treated with nintedanib.

Acknowledgements: Medical writing assistance, supported financially by Boehringer Ingelheim, was provided by Julie Fleming and Wendy Morris (FleishmanHillard Fishburn, London, UK) during the preparation of this article. The authors were fully responsible for all content and editorial decisions, were involved at all stages of manuscript development, and have approved the final version of the manuscript, which reflects the authors' interpretation and conclusions.

Support statement: The TOMORROW and INPULSIS trials were funded by Boehringer Ingelheim. Funding information for this article has been deposited with the Crossref Funder Registry.

Conflict of interest: I. Noth reports personal fees for consultancy, lecturing, advisory board work and travel support from Boehringer Ingelheim and Roche/Genentech, personal fees for advisory board work from Sanofi and Global Blood Therapeutics, and personal fees for consultancy from Veracyte, outside the submitted work. M. Wijsenbeek reports grants and other from Boehringer Ingelheim and Roche, and other from Galapagos, outside the submitted work. M. Kolb reports grants from Canadian Pulmonary Fibrosis Foundation and Canadian Institute for Health Research, has acted as site principal investigator in industry-sponsored clinical trials for Roche, Sanofi and Boehringer Ingelheim, grants from and advisory board work for Pulmonary Fibrosis Foundation, personal fees for advisory board work from Boehringer Ingelheim, GlaxoSmithKline, AstraZeneca, Vertex, Genoa, Gilead, Prometic and Alkermes, and grants and personal fees for advisory board work from Roche Canada and Janssen, outside the submitted work. F. Bonella reports personal fees for consultancy, lecturing and travel support from Boehringer Ingelheim and Roche, outside the submitted work. L. Moros is an employee of Boehringer Ingelheim. D. Wachtlin is an employee of Boehringer Ingelheim. T.J. Corte reports grants and personal fees for travel, lecturing and advisory board compensation from Boehringer Ingelheim, grants and personal fees for travel, lecturing, steering committee work and advisory board compensation from Roche, grants from Gilead, Bayer, Intermune and BMS, and personal fees for advisory board compensation from AstraZeneca, during the conduct of the study.

\section{References}

1 Raghu G, Collard HR, Egan JJ, et al. An official ATS/ERS/JRS/ALAT statement: idiopathic pulmonary fibrosis: evidence-based guidelines for diagnosis and management. Am J Respir Crit Care Med 2011; 183: 788-824.

2 Collard HR, Ward AJ, Lanes S, et al. Burden of illness in idiopathic pulmonary fibrosis. J Med Econ 2012; 15: 829-835.

3 Hyldgaard C, Hilberg O, Bendstrup E. How does comorbidity influence survival in idiopathic pulmonary fibrosis? Respir Med 2014; 108: 647-653.

4 Raghu G, Amatto VC, Behr J, et al. Comorbidities in idiopathic pulmonary fibrosis patients: a systematic literature review. Eur Respir J 2015; 46: 1113-1130.

5 Kreuter M, Ehlers-Tenenbaum S, Palmowski K, et al. Impact of comorbidities on mortality in patients with idiopathic pulmonary fibrosis. PLoS One 2016; 11: e0151425.

6 Kreuter M, Costabel U, Richeldi L, et al. Statin therapy and outcomes in trials of nintedanib in idiopathic pulmonary fibrosis. Respiration 2018; 95: 317-326.

7 Piepoli MF, Hoes AW, Agewall S, et al. 2016 European Guidelines on cardiovascular disease prevention in clinical practice: The Sixth Joint Task Force of the European Society of Cardiology and Other Societies on Cardiovascular Disease Prevention in Clinical Practice (constituted by representatives of 10 societies and by invited experts). Developed with the special contribution of the European Association for Cardiovascular Prevention \& Rehabilitation (EACPR). Eur Heart J 2016; 37: 2315-2381.

8 Richeldi L, du Bois RM, Raghu G, et al. Efficacy and safety of nintedanib in idiopathic pulmonary fibrosis. $N$ Engl J Med 2014; 370: 2071-2082.

9 Hilberg F, Roth GJ, Krssak M, et al. BIBF 1120: triple angiokinase inhibitor with sustained receptor blockade and good antitumor efficacy. Cancer Res 2008; 68: 4774-4782.

10 Wollin L, Maillet I, Quesniaux V, et al. Antifibrotic and anti-inflammatory activity of the tyrosine kinase inhibitor nintedanib in experimental models of lung fibrosis. J Pharmacol Exp Med 2014; 349: 209-220.

11 Shah RR, Morganroth J. Update on cardiovascular safety of tyrosine kinase inhibitors: with a special focus on QT interval, left ventricular dysfunction and overall risk/benefit. Drug Saf 2015; 38: 693-710.

12 Corte T, Bonella F, Crestani B, et al. Safety, tolerability and appropriate use of nintedanib in idiopathic pulmonary fibrosis. Respir Res 2015; 16: 116.

13 Boehringer Ingelheim Pharmaceuticals, Inc. OFEV (nintedanib). Prescribing information. 2018. https://docs. boehringer-ingelheim.com/Prescribing\%20Information/PIs/Ofev/ofev.pdf Date last accessed: August 16, 2018.

14 Boehringer Ingelheim. OFEV (nintedanib). Summary of product characteristics. 2018. https://www.ema.europa.eu/ en/documents/product-information/ofev-epar-product-information_en.pdf Date last accessed: August 16, 2018.

15 Richeldi L, Costabel U, Selman M, et al. Efficacy of a tyrosine kinase inhibitor in idiopathic pulmonary fibrosis. N Engl J Med 2011; 365: 1079-1087.

16 Crestani B, Quaresma M, Kaye M, et al. Long-term nintedanib treatment in idiopathic pulmonary fibrosis (IPF): new data from INPULSIS-ON. Eur Respir J 2017; 50: OA3402. 
17 Bonella F, Kreuter M, Hagmeyer L, et al. Insights from the German compassionate use program of nintedanib for the treatment of idiopathic pulmonary fibrosis. Respiration 2016; 92: 98-106.

18 Hughes G, Toellner H, Morris H, et al. Real world experiences: pirfenidone and nintedanib are effective and well tolerated treatments for idiopathic pulmonary fibrosis. J Clin Med 2016; 5: 78.

19 Galli JA, Pandya A, Vega-Olivo M, et al. Pirfenidone and nintedanib for pulmonary fibrosis in clinical practice: tolerability and adverse drug reactions. Respirology 2017; 22: 1171-1178.

20 Noth I, Oelberg D, Kaul M, et al. Safety and tolerability of nintedanib in patients with idiopathic pulmonary fibrosis in the USA. Eur Respir J 2018; 52: 1702106.

21 Toellner H, Hughes G, Beswick W, et al. Early clinical experiences with nintedanib in three UK tertiary interstitial lung disease centres. Clin Transl Med 2017; 6: 41.

22 Brunnemer E, Wälscher J, Tenenbaum S, et al. Real-world experience with nintedanib in patients with idiopathic pulmonary fibrosis. Respiration 2018; 95: 301-309. 